\title{
Molecular genetic data favoring a sequential clonal transformation of a large $B$ cell lymphoma into an anaplastic large T cell lymphoma, ALK-negative
}

\author{
Tomas Vanecek ${ }^{1} \cdot$ Kimberly Walker $^{2} \cdot$ Linden L Watson $^{2}$ • Arundhati Rao ${ }^{2}$. \\ Debby Rampisela $^{2} \cdot$ Ludvik R. Donner ${ }^{2}$
}

Received: 3 March 2015 / Accepted: 26 May 2015 /Published online: 13 June 2015

(C) Springer-Verlag Berlin Heidelberg 2015

\begin{abstract}
A large B cell lymphoma with monoclonal rearranged, sequentially identical TRG and near identical IGH genes twice relapsed as an anaplastic large $\mathrm{T}$ cell lymphoma, ALK-negative. While these IGH and TRG rearrangements were also detected in both relapsed lymphomas, near identical IGK rearrangement was present in the primary and the first relapse, though not in the second relapse, and identical TRB rearrangement was detected only in both relapses, not in the primary. Normal T lymphocytes were the only $\mathrm{T}$ cell component found in the primary lymphoma, thus excluding a possibility that the primary lymphoma was a classic composite $\mathrm{B}$ and $\mathrm{T}$ cell lymphoma. Our data, particularly, the presence of sequentially identical monoclonal TRG and near identical IGH rearrangement in the primary and both relapsed lymphomas, favor a gradual transformation of a B cell lymphoma into a $\mathrm{T}$ cell lymphoma. In our opinion, this previously not described phenomenon likely represents a composite lymphoma in statu nascendi.
\end{abstract}

Keyword Large B cell lymphoma · T lymphocytes · IGK rearrangement

Ludvik R. Donner

ldonner@sw.org

1 Sikl's Department of Pathology, School of Medicine, Charles University, Pilsen, Czech Republic

2 Department of Pathology, Scott \& White Healthcare, The Texas A\&M Health Science Center College of Medicine, 2401 South 31st Street, Temple, TX, USA

\section{Introduction}

Rare cases of composite lymphoma composed of neoplastic B cell and $T$ cells [1-8] as well as simultaneous or sequential development of $\mathrm{B}$ cell and $\mathrm{T}$ cell lymphoma in the same patient $[9,10]$ point toward the possibility that rare lymphomas undergo lineage infidelity with resulting clonal development of a T cell lymphoma from a B cell lymphoma and vice versa. A recent large flow cytometry study revealed that lymphomas composed of neoplastic B and T cells constituted mere $0.4 \%$ of all studied lymphomas [7]. Due to technical limitations, this development has so far not been sufficiently analyzed. Molecular genetic analysis of the case that we present in this article delineates a sequential transformation of a B cell lymphoma into a T cell lymphoma. A similar case was not, to the best of our knowledge, previously described.

\section{Case report}

A 53-year-old white man presented for evaluation of a retroperitoneal mass that was detected in April 2008 in another hospital. A confluent paraortal and left inguinal adenopathy was confirmed by computed tomography. Six cycles of CHOP-Rituxan therapy (dexamethasone, doxorubicin, vincristine, cyclophosphamide, and rituximab) resulted in complete remission. He developed a relapse in the form of a right axillary lymphadenopathy and moderate to marked splenomegaly in February 2011. Five cycles of salvage ICE chemotherapy (ifosfamide, carboplatin, etoposide) led to remission. He developed a second relapse (generalized lymphadenopathy and progressive hepatosplenomegaly) in July 2012. His course became complicated by neutropenic fever, respiratory distress, and encephalopathy. He expired a few weeks later. Generalized lymphadenopathy, marked splenomegaly, 
extensive involvement of portal tracts, and multifocal lymphomatous involvement of the kidneys and bone marrow were found during autopsy.

\section{Materials and methods}

Immunohistochemistry Immunohistochemical staining was performed on formalin-fixed, paraffin-embedded tissue sections. Antibodies against the following epitopes were used: CD2, CD4, CD5, CD7, CD8, CD15, CD30, CD43, CD45, CD45RO, CD138, MUM1, EMA, ALK-1, cyclin D1 (Dako, Carpinteria, CA, USA), CD10, CD20, CD22, CD79a, bcl-2, bcl-6, Pax-5, BOB.1, OCT-2, Ki-67, TdT (Leica/Novocastra Microsystems, Buffalo Grove, IL, USA), CD163, EBVLMP1 (Cell Marque, Rocklin, CA, USA), CXCL13 (R\&D, Minneapolis, MN, USA), and C-MYC (Ventana Medical Systems, Tucson, AZ, USA). The antibodies were monoclonal except for the antibody against CXCL13 that was polyclonal. The slides to be immunostained for CD15, CD20, CD4, CD79a, CXCL13, and Ki-67 were treated by EnVision $^{\mathrm{TM}}$ FLEX Target retrieval solution low pH (Dako), and the other slides were treated by EnVision ${ }^{\text {TM }}$ FLEX Target retrieval solution high $\mathrm{pH}$ (Dako).

Flow cytometry The following antibodies were utilized for flow cytometry analysis: anti-CD2, CD3, CD4, CD5, CD7, CD8, CD10, CD16, CD19, CD20, CD23, CD30, CD33, CD56, FMC7, kappa, lambda, TCR $\alpha / \beta$, and TCR $\gamma / \delta$ (Becton-Dickinson, San Jose, CA, USA).

Molecular genetics The analysis was performed in two laboratories, Temple and Pilsen. The DNA was extracted from formalin-fixed, paraffin-embedded tissue using the Qiagen QIAamp Mini Kit (Qiagen, Germantown, MD, USA) according to the manufacturer's protocols in the Temple laboratory and NucleoSpin Tissue Kit (Macherey Nagel, Puren, Germany) in the Pilsen laboratory. In the Temple laboratory, the DNA was amplified by Invivoscribe Technologies (San Diego, CA, USA) kits targeting IGH, IGK, IGL, TRG, and TRB. The amplicons were detected on the ABI 3500 genetic analyzer (Life Technologies) using differential fluorescence technology. Integrity of DNA was verified in the Pilsen laboratory by PCR with BIOMED-2 control primer set (Generi Biotech, Hradec Kralove, Czech Republic) amplifying 100-600-bp range of fragments. The clonality analysis of TRG and IGH, and IGK and IGL was performed there with BIOMED-2 multiplex PCR system followed by fragment analysis of amplicons on ABI3130xI Genetic analyzer (Life Technologies) [11]. Appropriate positive, negative, and contamination controls as well as the control ladder were run alongside the patient sample in both laboratories. Amplicons with one or two prominent peaks within the valid size range were considered to be monoclonal. The monoclonal peaks were re-amplified in the Pilsen laboratory with unlabeled "peak"-specific primer sets and purified and both strands sequenced using Bid Dye Terminator Sequencing kit (Life Technologies). The sequences were compared using in silico algorithms for comparing IG and TR nucleotide sequences $[12,13]$.

Deep sequencing for TRG and IGH was performed in the Temple laboratory using LymphoTrack ${ }^{\circledR}$ IGH and TRG assays (Invivoscribe Technologies, Carlsbad, CA) on the Ion Torrent PGM (Life Technologies). Multiplex master mix for IGH targets the conserved framework
Fig. 1 The primary lymphoma. The large neoplastic cells are CD20-positive and have high proliferation rate. Only scanty $\mathrm{T}$ lymphocytes are present. a $\mathrm{H} \& \mathrm{E}$. b CD20. c CD3. d Ki-67. Original magnification: $\mathbf{a}=\times 100$,

b- $\mathbf{d}=\times 40$

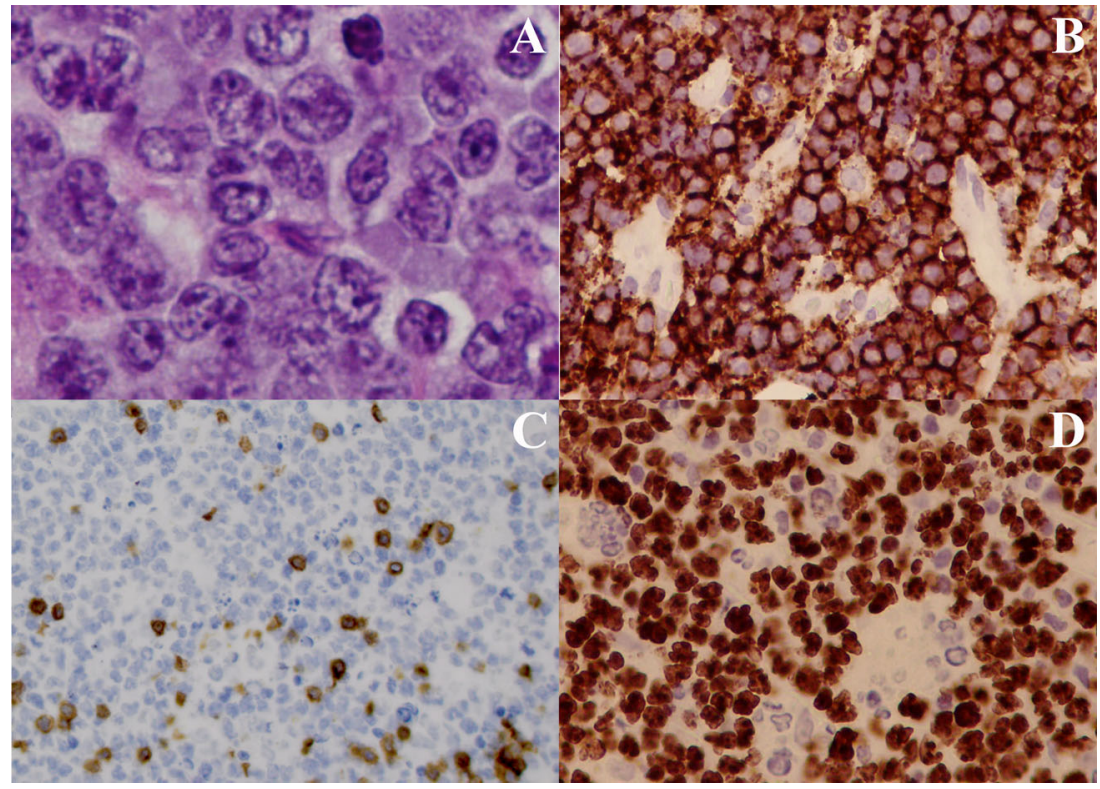


region 1 (FR1) within the $\mathrm{VH}$ and the $\mathrm{JH}$ regions, while multiplex master mix for TRG targets all conserved regions within the $\mathrm{V}$ and the $\mathrm{J}$ gene regions described in lymphoid malignancies. Primers included in the master mixes are designed with Life Technologies (Thermo Fisher) adapters and contain 12 different indices per kit. After routine DNA extraction and PCR amplification with primers and barcode adaptors onto the Ion Sphere Particles (ISPs), template-positive ISPs were subjected to next-generation sequencing (NGS). This was performed on Ion 316 chips using the Ion PGM $^{\mathrm{TM}}$ Sequencing 200 kit v2 and the Ion Torrent PGM (Life Technologies). NGS data were analyzed using the LymphoTrack PGM Software package, and $\mathrm{V}-\mathrm{J}$ sequence frequency plots as well as relative $\mathrm{V}-\mathrm{J}$ usage plots were presented.

\section{Results}

\section{Pathologic study}

The parenchyma of the left inguinal lymph node excised in May 2008 was replaced by large blasts with round nuclei, a few nucleoli, and scanty moderately basophilic cytoplasm. Starry sky pattern was prominent. The neoplastic cells were diffusely positive for CD20, CD79a, Pax-5, bcl-6, BOB.1, and OCT-2 and were negative for CD10, bcl-2, CD15, CD30, CD43, MUM1, TdT, and EBV-LMP1 by immunohistochemistry. The C-MYC was moderately expressed in $40 \%$ of neoplastic cells. Sixty percent of the cells were Ki-67-positive. The neoplastic cells were positive for CD19, CD20, and FMC7 and negative for CD5, CD10, CD23, and CD56 by

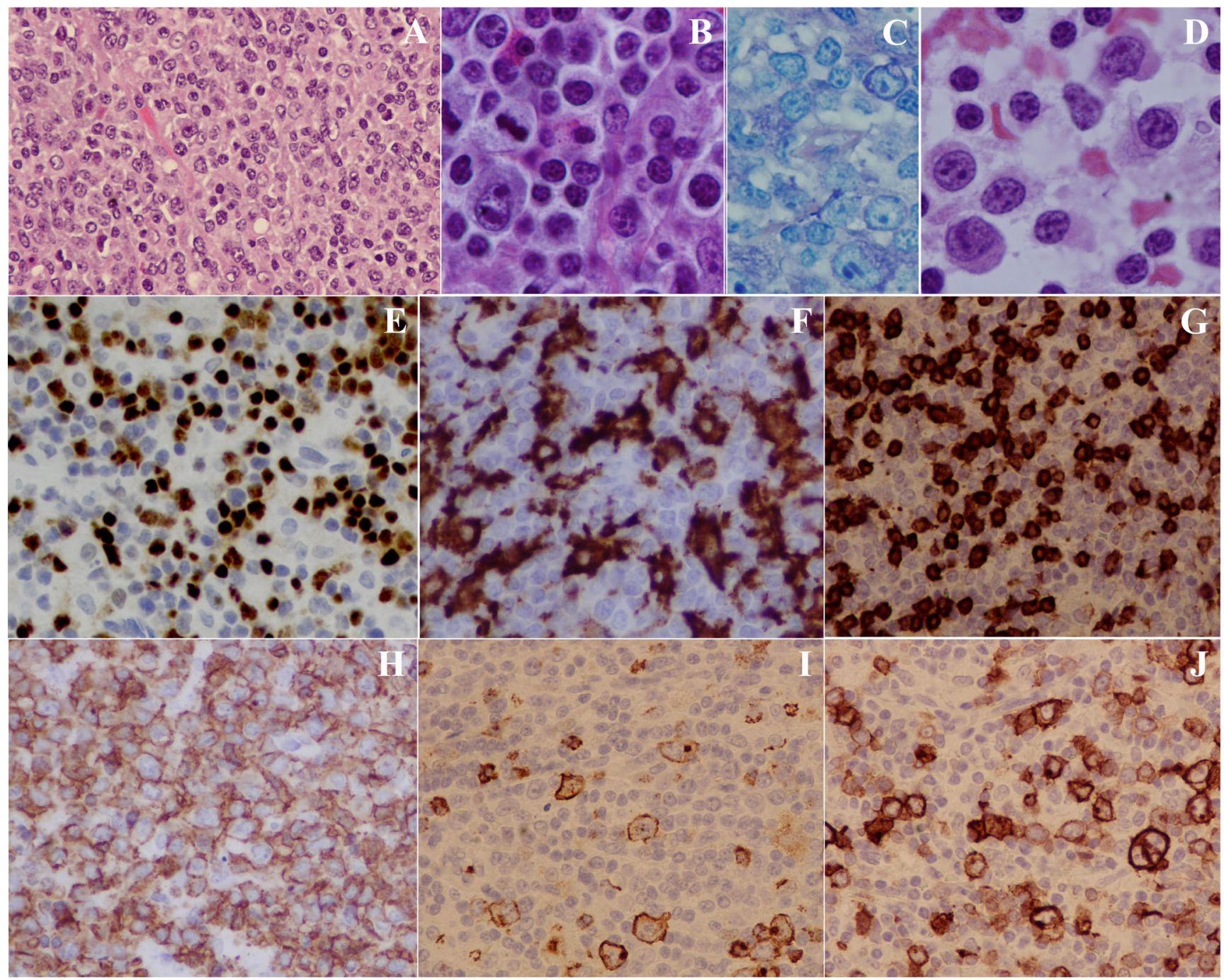

Fig. 2 The first relapsed lymphoma. Single cells and tiny foci of neoplastic T cells with large round nuclei, dispersed chromatin, large nucleoli, and scanty basophilic cytoplasm are present in polymorphous background that contains many B cells, T cells, histocytes as well as plasma cells, and eosinophils. a Lower power view. b-d Foci of neoplastic cells (a, b H\&E, c Giemsa stain, d smear). e Pax-5. f CD163. g CD3. h CD45RO. i CD15. j CD30. Original magnification: $\mathbf{a}, \mathbf{e}-\mathbf{j}=\times 40, \mathbf{b}-\mathbf{d}=\times 100$ 
flow cytometry analysis. The T cells constituted $15 \%$ of the lymphoid cells and were immunophenotypically normal. They expressed CD2, CD3, CD5, and CD7 and had a normal helper to suppressor ratio of 2:1. Additionally, several 3- $\mu \mathrm{m}$ thick recuts were re-examined and were immunostained for CD3. All T cells were mature lymphocytes. No atypical T cells were found. The neoplasm was classified as a large B cell lymphoma (Fig. 1).

The second specimen, the right axillary lymph node with relapsed lymphoma that was excised in March 2011, was very different and was diagnostically very challenging. Its parenchyma contained many clusters of CD163-positive histiocytes, numerous $\mathrm{T}$ lymphocytes, foci of uninvolved lymph node parenchyma with lymphoid follicles, many eosinophils, and occasional plasma cells. Scattered were large blasts with round or mildly lobulated nuclei, fine chromatin, prominent nucleoli, and scanty moderately basophilic cytoplasm. They were positive for $\mathrm{CD} 2, \mathrm{CD} 3, \mathrm{CD} 30, \mathrm{CD} 43, \mathrm{CD} 45, \mathrm{CD} 45 \mathrm{RO}$, and MUM1, a subset of them was positive for CD15, and they were negative for CD4, CD5, CD7, CD8, CD19, CD20, CD22, CD56, CD79a, CD138, Pax-5, bcl-6, EMA, BOB.1, OCT-2, CXCL13, EBV-LMP1, and ALK-1. Fifty percent of the cells were Ki-67-positive. The C-MYC was expressed weakly in only $8 \%$ of neoplastic cells. Forty percent of the cells were TCR $\alpha / \beta$-positive by flow cytometry (Fig. 2).

We were unable to classify this lymphoma prior to receiving a subsequent lymph node that was biopsied in July 2012 from the right inguinal region. The lymph node parenchyma was effaced by diffuse lymphoid infiltrate composed of numerous large blasts, many $\mathrm{T}$ lymphocytes, and numerous
CD163-positive histiocytes. The number of blasts was, in comparison to the previously biopsied lymph node, markedly increased. They were positive for CD2, CD3, CD30, CD45, CD45RO, and MUM1 and were negative for CD4, CD5, CD7, CD8, CD10, CD15, CD19, CD20, CD22, CD79a, ALK-1, bcl-6, BOB.1, cyclin D1, CXCL13, EMA, and Pax5. The C-MYC was expressed in $10 \%$ of neoplastic cells, and its expression was weak to moderate. These findings were confirmed and expanded by flow cytometry analysis. The neoplastic cells were expressing CD2, CD3, CD30, and $\mathrm{TCR} \alpha / \beta$ but not CD4, CD5, CD7, CD8, CD10, CD19, CD20, CD22, CD23, CD56, FMC7, and TCR $\gamma / \delta$. The B cells constituted $1-2 \%$ of the population and were all mature $\mathrm{B}$ lymphocytes. They were $\mathrm{CD} 19+, \mathrm{CD} 20+, \mathrm{CD} 10^{-}, \mathrm{K}-$, and $\lambda$ - by flow cytometry and CD19+, CD20+, CD22+, CD79a+, and Pax-5+ by immunohistochemistry. No B cell blasts were found (Fig. 3). The lymphoma in the both relapses was classified as anaplastic large T cell lymphoma, ALK-negative. It was emerging, in polymorphous background, during the first relapse and became established in the second relapse.

\section{Molecular genetic study—polymerase chain reaction/clonal analysis}

The IGH and TRG genes were monoclonally rearranged in the primary and both relapses. The monoclonal rearrangement of the IGH gene was detected only by deep sequencing. When analyzed in the Pilsen laboratory, two monoclonal TRG peaks, 218 and $243 \mathrm{bp}$, were found in the primary and both relapses. It appears that rearrangement of the same TRG region was
Fig. 3 The second relapsed lymphoma. In contrast to the first relapse, the neoplastic T cells are predominant. Note that the $\mathrm{B}$ cells are very scanty. a-c $H \& E$ (c smear). d CD2. e CD30. f CD45RO. g Pax-5. Original magnification: a, $\mathbf{d}-\mathbf{g}=\times 40$, $\mathbf{b}$, $\mathbf{c}=\times 100$

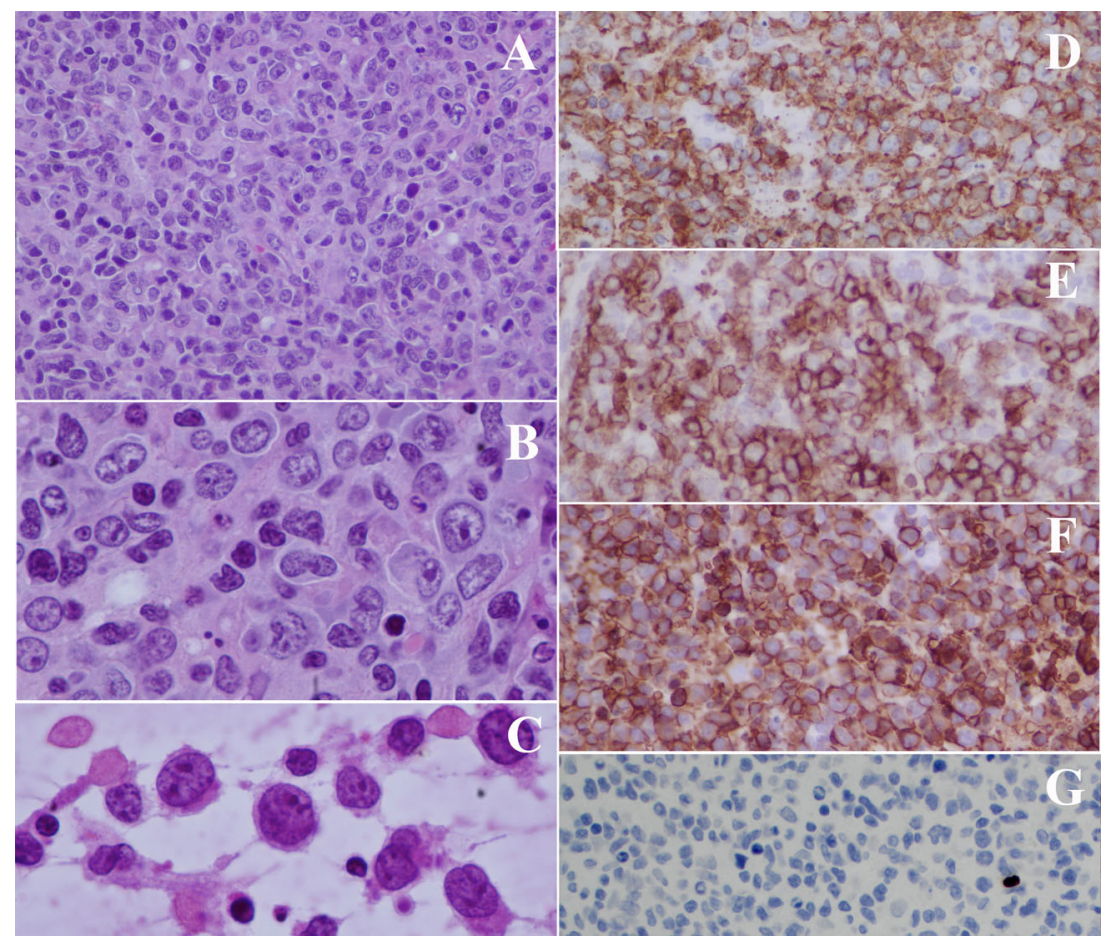


detected in both laboratories. The 218-bp peak was found in the Vglf-Jg1.3_2.3 region (Vg2-8-J1/J2). The 243-bp peak, found in the Vglf-Jg1.1 2.1 region(Vg2-8-JP1/JP2), resulted from rearrangement of Vg4 and JP2 segments. The 218-bp
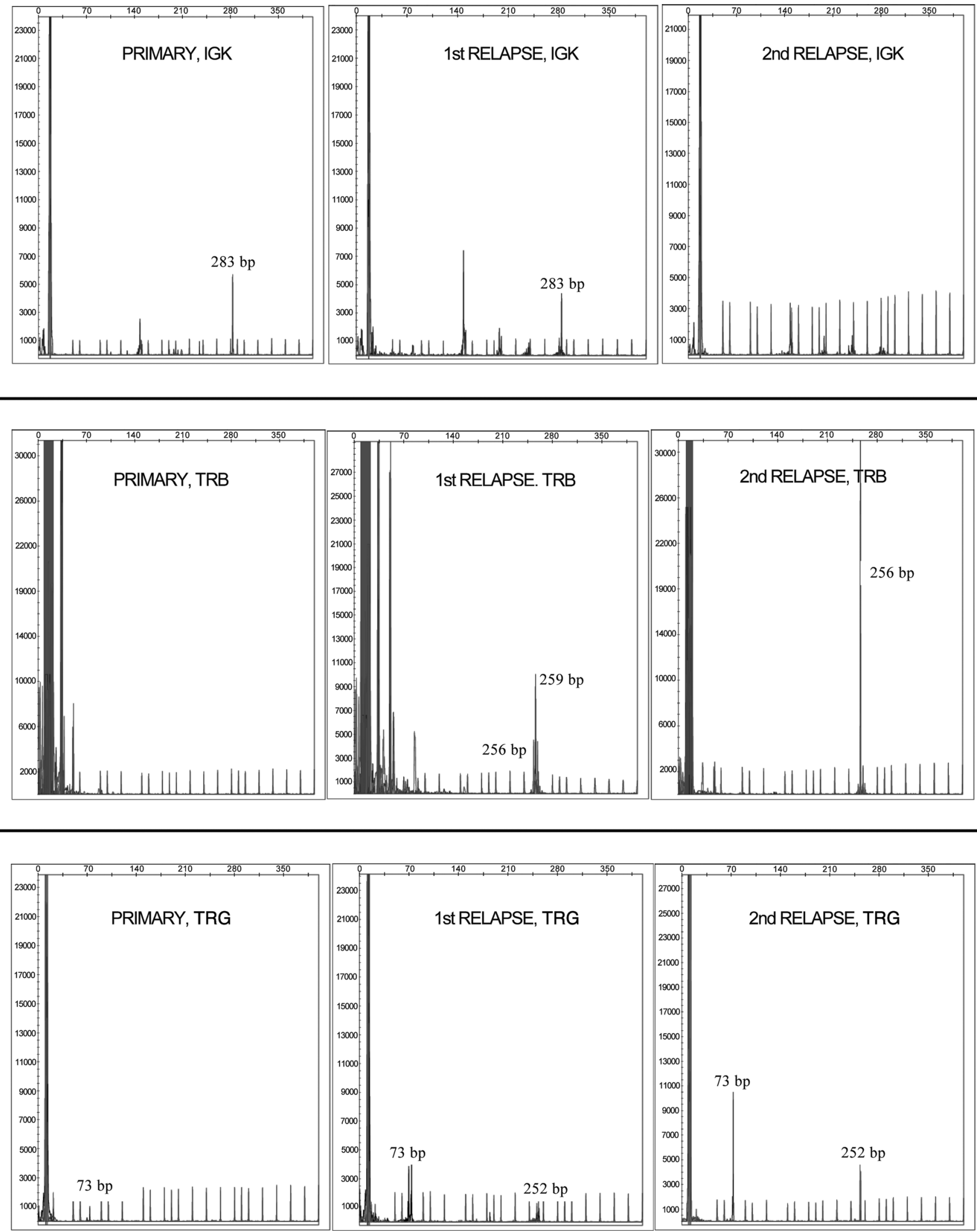

Fig. 4 Polymerase chain reaction analysis of the IGK, TRB, and TRG (Temple laboratory study). The monoclonal amplicons are marked by base pairs 
peak was sequenced only from the second relapsed lymphoma and represents a nonproductive allele that most probably arose during bi-allelic TRG rearrangement [14]. When analyzed in the Temple laboratory, a 73-bp TRG peak was found in the primary and both relapses and both relapses also contained an additional 252-bp monoclonal peak. The 73-bp peak was also detected in a $4-\mathrm{mm}^{2}$ laser capture microdissected section of the primary that contained approximately 5600 cells $(840 \mathrm{~T}$ lymphocytes). The 73-bp peak was low in the primary, became stronger in the first relapsed, and strong in the second relapsed lymphomas. The 73-bp peak represents the Vg1-8,9$\mathrm{Jg} 1 / 2$, and the 252 peak represents the prior Alt. $\mathrm{Vg}-\mathrm{Jg} 1 / 2$ regions of the TRG.

The IGK was monoclonally rearranged in the primary and the first relapsed lymphoma, but this rearrangement was lost in the second relapse. The monoclonal peak, measured $282 \mathrm{bp}$ in the Pilsen laboratory and $283 \mathrm{bp}$ in the Temple laboratory, represents the $\mathrm{Vk} 3 /$ intron-Kde region of the IGK gene. The TRB was studied only in the Temple laboratory. Its
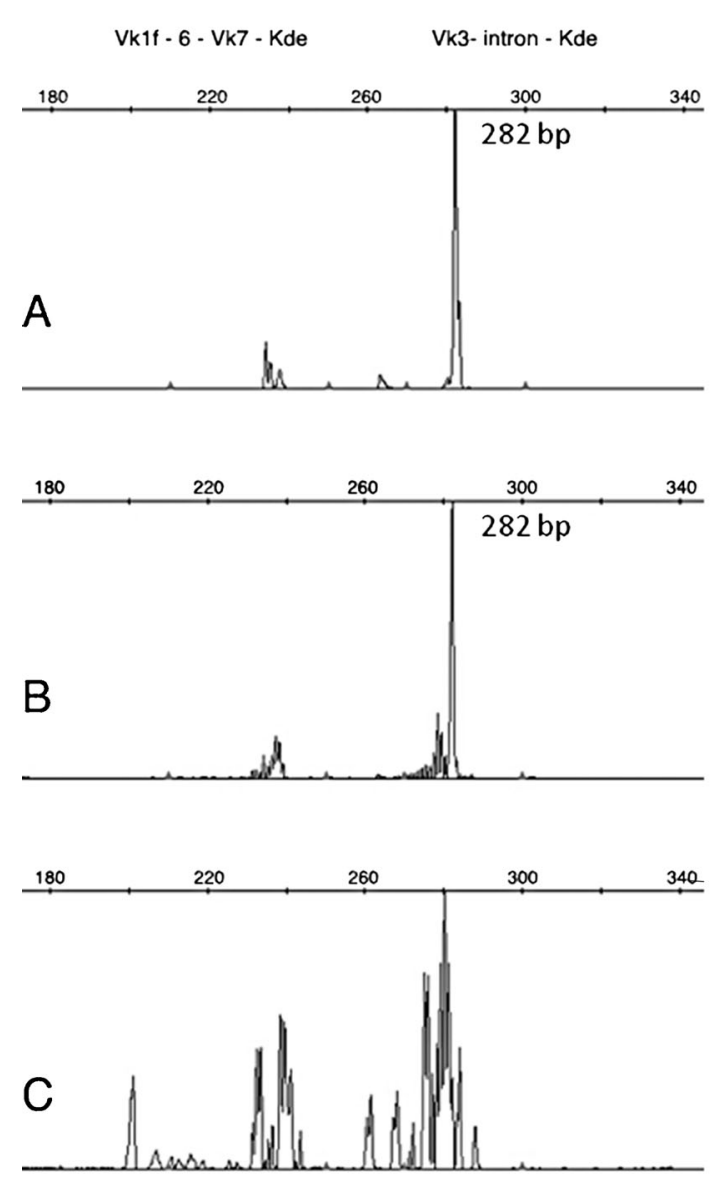

Fig. 5 Polymerase chain reaction analysis of the IGK and TRG (Pilsen laboratory study). A 282-bp monoclonal peak is in Vk3/intron-Kde amplification region of the primary (a) and the first relapsed lymphomas (b), while there is only a polyclonal pattern of the gene in the second relapsed lymphoma (c). A 243-bp TRG monoclonal peak is in monoclonal 256-bp peak was detected in both relapsed lymphomas but not in the primary lymphoma. The first relapse also contained a 259-bp monoclonal peak. The 256-bp peak represents the $\mathrm{V} \beta-\mathrm{J} \beta$ region, and the 259 -bp peak also represents the $V \beta-J \beta$ region of the TRB (Figs. 4 and 5). The status of the IGL in the primary and both relapses was analyzed in the Temple laboratory. No rearrangement was identified.

Sequencing of the TRG and IGK amplicons was performed in the Pilsen laboratory, and the sequences there were compared by IgBLAST and BLAST [12, 13]. The 243-bp TRG peak remained sequentially identical in the primary and both relapsed lymphomas and arose from the TRGV4*01/ TRGJP2*01 junction. The 218 -bp peak of the second relapse revealed joining of the TRGV $2 * 02$ and of the TRGJ $1 * 02$ or TRGJ $1 * 01$ or TRGJ $2 * 01$ segment.

The IGK 282-bp peak in the primary and the first relapse arose from the IGKV3-20*01/Kde junction. In comparison to the germ line control, the primary and the first relapsed lymphomas differed in $4 \mathrm{bp}$ each, but they had only one of the

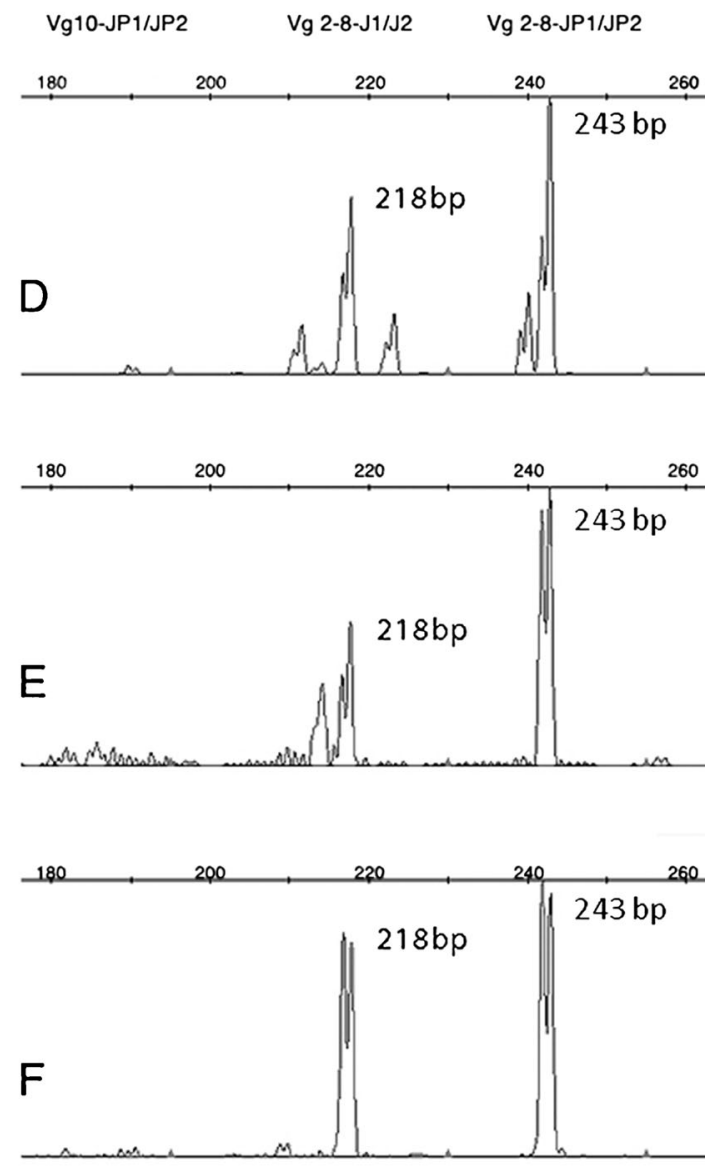

Vg2-8-JP1/JP2 amplification region of the primary and both relapsed lymphomas. Several other minor peaks, including that of $218 \mathrm{bp}$, are also detected. d Primary lymphoma. e First relapsed lymphoma. f Second relapsed lymphoma 
substitutions in common (Fig. 6). The Vk3/intron-Kde transition region of the IGK gene of the primary and the first relapsed lymphomas was repeatedly sequenced, and the results were almost identical. Because IGK-DE rearrangements do not undergo somatic hypermutations, we interpret the substitution of total of 4 bases to be likely artifactual, caused by formalin cross-linking and difference in tissue preservation $[14,15]$.

\section{Deep sequencing}

The LymphoTrack read counts varied from 3000 to 47,150 for the most commonly identified sequences, and the coverage ranged from 69.2 to $99.55 \%$. The length of the most common sequences ranged from 148 to $220 \mathrm{bp}$. The clone identified by IGH sequencing was VH1. The cells containing this clone constituted $60 \%$ of the population in the primary with successive decrease to $20 \%$ of the cells in the first relapsed and $8 \%$ in the second relapsed lymphomas. There was also an oligoclonal pattern in the first relapse and a polyclonal pattern in the second relapse. The IGH clone was sequentially identical in the primary and both relapses except for a single additional nucleotide in the second relapse. The TRG clone was Vg4-JgP2. It was detected in $15 \%$ of the cells in the primary, less than $2 \%$ of the cells in the first relapse, and $50 \%$ of the cells in the second relapse. The primary and the first relapsed lymphomas also contained polyclonal background. The TRG clone was sequentially identical in the primary and both relapses (Fig. 7a-g).

\section{Discussion}

Presence of identical clonal peak of TRG and near identical clonal peak of IGH in the primary large B cell lymphoma and both relapses, a decrease in intensity of the IGH peaks in both relapses that was coinciding with an increase in the TRG peak in the second relapse, presence of clonally rearranged TRB only in both relapses, and a loss of monoclonal IGK peak in the second relapse seem to indicate that the transformation of B cell lymphoma into T cell lymphoma was a gradual process. Sequentially identical TRG and near identical IGH monoclonal rearrangement in all three lymphomas provides the strongest argument in favor of their clonal relatedness. The number of $\mathrm{T}$ cells in the primary was estimated by flow cytometry to represent $15 \%$ of the cells. Because presence of at least $5 \%$ of cells with monoclonal TRG rearrangement is necessary for their detection by polymerase chain reaction in our laboratory, at least a third of the $840 \mathrm{~T}$ cells expected to be present in the microdissected portion would have to be positive for the rearrangement and to be, by implication, morphologically abnormal. Thus, the failure to detect a neoplastic $\mathrm{T}$ cell component

TRG, 243 bp peak

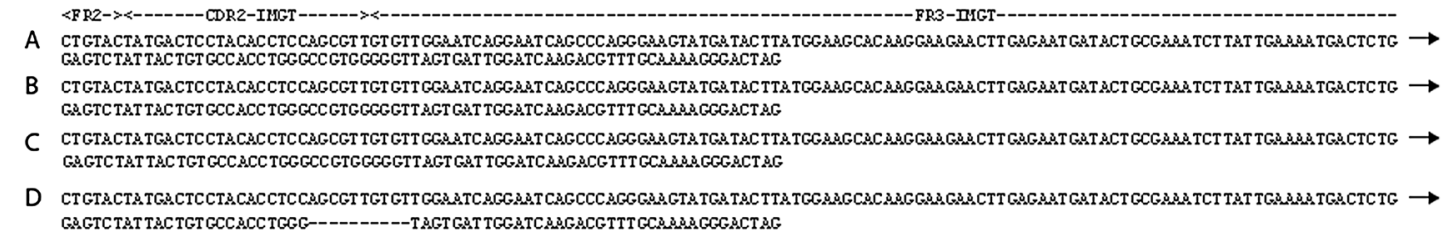

TRG, 218 bp peak

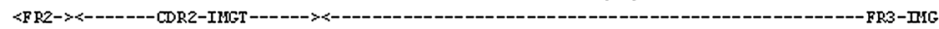

E cagtactatgactcctaca.cTcCa.

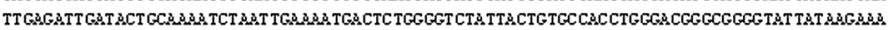

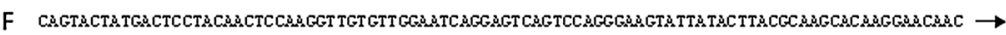

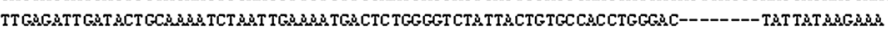

IGK, 282 bp peak

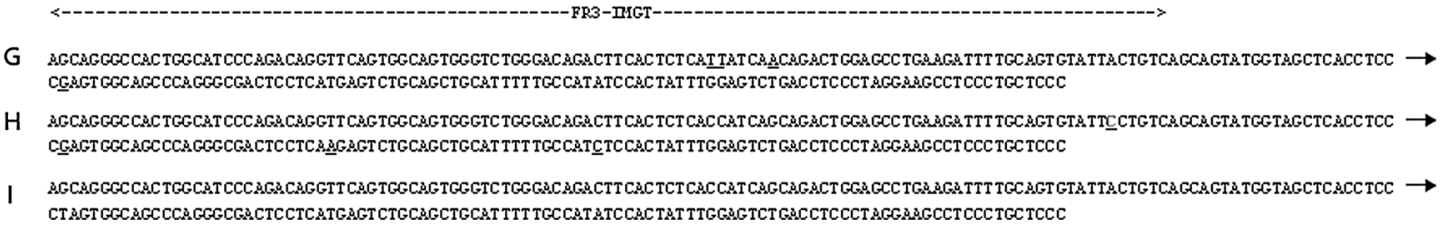

Fig. 6 Nucleotide sequence of TRG 243- and 218-bp peaks and IGK 282-bp peak. a Primary lymphoma 243 bp. b First relapsed lymphoma 243 bp. c Second relapsed lymphoma 243 bp. d Germ line control (TRGV4*01/TRGJP $2 * 01$ ). e Second relapsed lymphoma 218 bp. f Germ line control (TRGV2*02/TRGJ1*02, TRGJ1*01, TRGJ2*01). Note that the sequences of the TRG 243-bp peak in the primary and both relapsed lymphomas are identical. The sequences of the 218-bp peak are non-productive. g Primary lymphoma $282 \mathrm{bp}$. h First relapsed lymphoma 282 bp. i Germ line control (IGKV3-20*01+Kde). Discrepancies between nucleotides in the primary and the first relapse of the IGK 282-bp peak are underlined 
A

Deep sequencing. IGH and TRG Sequences.

IGH. VH1 peak.

Primary lymphoma

CTTCTGGATACACCTTCAGCGACTACCATATATAGCAACTTTGACAGGCCCCT

AGACAAGGGCTTGGGTGAATGGGCTGGCTCAACCCTTATAGTGGTGGCACAA

ACTCTGCACAGAAATTTCAGGCTGGGTCACCATGACCAGGGACACGTCCGAC GACACGGCCTAAAAATATGGGGCAGGGAACTCT

$1^{\text {st }}$ relapsed lymphoma

CTTCTGGATACACCTTCAGCGACTACCATATATAGCAACTTTGACAGGCCCCT

AGACAAGGGCTTGGGTGAATGGGCTGGCTCAACCCTTATAGTGGTGGCACAA ACTCTGCACAGAAATTTCAGGCTGGGTCACCATGACCAGGGACACGTCCGAC GACACGGCCTAAAAATATGGGGCAGGGAACTCT

\author{
$2^{\text {nd }}$ relapsed lymphoma \\ CTTCTGGATACACCTTCAGCGACTACCATATATAGCAACTTTGACAGGCCCCT \\ AGACAAGGGCTTGGGTGAATGGGCTGGCTCAACCCTTATAGTGGTGGCACAA \\ ACTCTGCACAGAAATTTCAGG \\ CGACACGGCCTAAAAATATGGGGCAGGGAACTCT
}

TRG. Vg4-JgP2 peak.

Primary lymphoma

GGAATCAGCCCAGGGAAGTATGATACTTATGGAAGCACAAGGAAGAACTTG

AGAATGATACTGCGAAATCTTATTGAAAATGACTCTGGAGTCTATTACTGTGC CACCTGGGCCGTGGGGGTTAGTGATTGGATCAAGACGTTTGCAA

\author{
1st relapsed lymphoma \\ GGAATCAGCCCAGGGAAGTATGATACTTATGGAAGCACAAGGAAGAACTTG \\ AGAATGATACTGCGAAATCTTATTGAAAATGACTCTGGAGTCTATTACTGTGC \\ CACCTGGGCCGTGGGGGTTAGTGATTGGATCAAGACGTTTGCAA \\ 2nd relapsed lymphoma \\ GGAATCAGCCCAGGGAAGTATGATACTTATGGAAGCACAAGGAAGAACTTG \\ AGAATGATACTGCGAAATCTTATTGAAAATGACTCTGGAGTCTATTACTGTGC \\ CACCTGGGCCGTGGGGGTTAGTGATTGGATCAAGACGTTTGCAA
}

Fig. 7 Deep sequencing analysis of the TRG and IGH genes. a IGH and TRG sequences. Note that a monoclonal TRG peak (Vg4-JgP2) is sequentially identical in the primary lymphoma and both relapses. A monoclonal IGH peak (VH1) is sequentially near identical in the primary lymphoma and both relapses (a single additional nucleotide in

in the primary B cell lymphoma by histology, immunohistochemistry, and flow cytometry does not support a possibility that this lymphoma contained two separate, fully developed, neoplastic components, a B cell lymphoma and a T cell lymphoma from the beginning and thus represented a classic composite B cell and T cell lymphoma. On the other hand, the data favor an early stage of development of composite lymphoma (composite lymphoma in statu nascendi). This conclusion is also supported by very low frequency of B lymphocytes and failure to detect any residual B cell lymphoma in the second relapse. It seems that the primary large B cell lymphoma contained a subpopulation of neoplastic B cells that were undergoing genetic/epigenetic changes representing early stage of transdifferentiation toward T cell lymphoma. These cells then continued the process of transdifferentiation, after the second relapse is underlined). b (b1-b6) $\mathrm{V}-\mathrm{J}$ sequence frequency plots with $\mathrm{V}-\mathrm{J}$ usage plots in insets. Monoclonal peaks are marked by arrows. b1 IGH primary lymphoma. b2 IGH first relapsed lymphoma. b3 IGH second relapsed lymphoma. b4 TRG primary lymphoma. b5 TRG first relapsed lymphoma. b6 TRG second relapsed lymphoma

eradication of original neoplastic B cells by chemotherapy, into $\mathrm{T}$ cell lymphoma.

Existence of clonal transformation of B cell lymphoma to $\mathrm{T}$ cell lymphoma is not necessarily surprising. It has been well established that under laboratory conditions, pro-B and pre-B cells can be reprogrammed into pluripotent stem cells and that even mature B cells can be converted into $\mathrm{T}$ cells by their dedifferentiation to uncommitted progenitors [16-18]. The chain of events that resulted in transformation of our case of large B cell lymphoma into anaplastic large $\mathrm{T}$ cell lymphoma is not known. It may be noteworthy that the Pax-5 gene, the essential regulator of B cell identity [19], was strongly expressed in the primary lymphoma but was not expressed in the relapsed lymphomas. We do not know whether this gene 


\section{B}
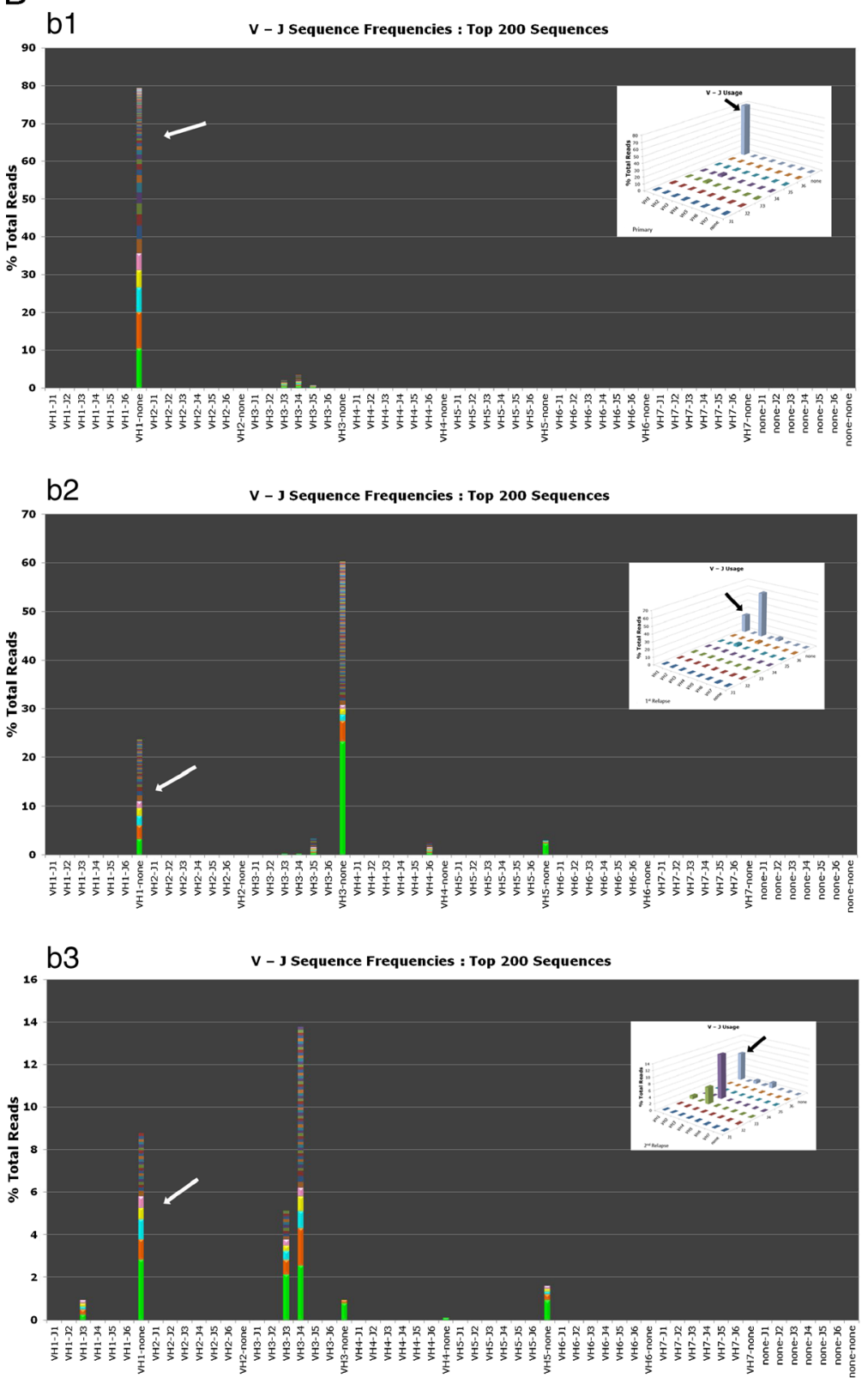

b4 $v$ - J Sequence Frequencies : Top 200 Sequences

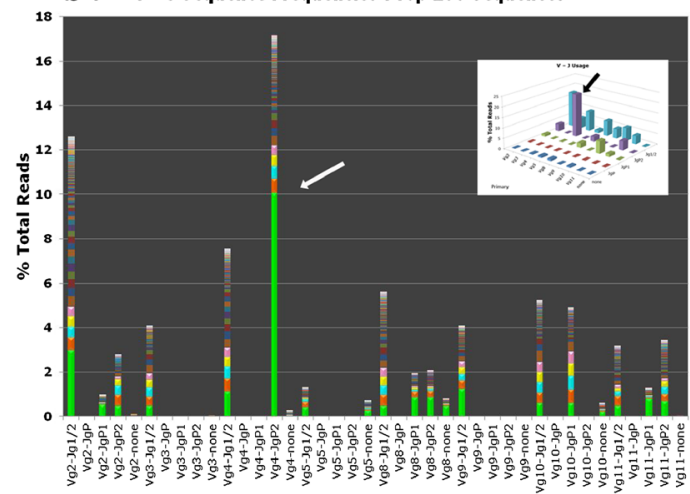

b5 $v$ - J Sequence Frequencies : Top 200 Sequences

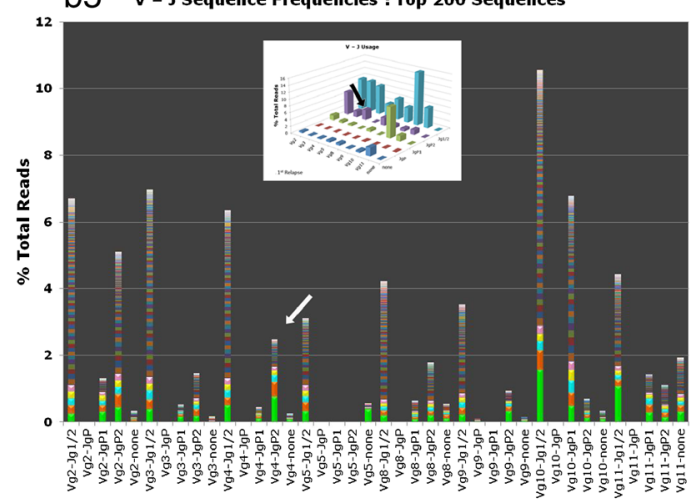

b6 $v$ - $\mathbf{u}$ Sequence Frequencies : Top 200 Sequences

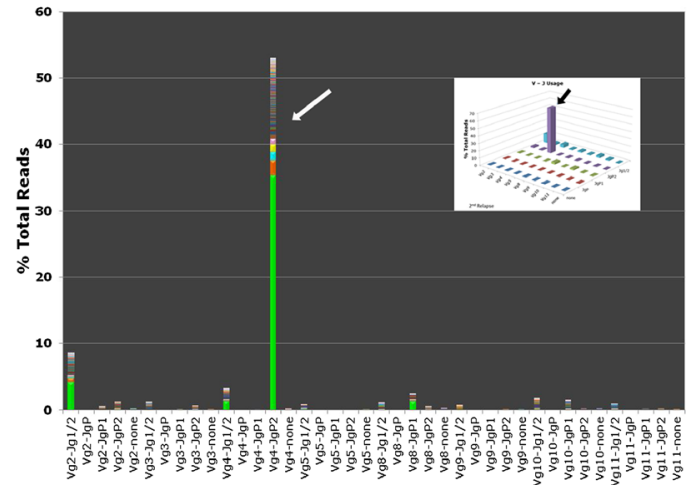

Fig. 7 (continued)

was lost or was epigenetically silenced in the recurrent lymphomas.

Although rare cases of clonal transdifferentiation of hematopoietic neoplasms were reported, their existence does not appear to be sufficiently recognized. They include transformation of lymphoid neoplasms into Langerhans cell neoplasms and histocytic sarcomas and chronic myeloid leukemia into $\mathrm{T}$ cell lymphoblastic lymphoma [20-25].

We were initially unable to classify the first relapsed lymphoma. Although we favored a peripheral T cell lymphoma, the presence of CD15-positive, CD30-positive blasts as well as background of numerous $\mathrm{T}$ lymphocytes, many eosinophils and occasional plasma cells were raising a possibility of
Hodgkin's lymphoma. However, absence of Sternberg-Reed cells was a strong argument against this interpretation. Several cases of CD30-positive $\mathrm{T}$ cell lymphomas coexpressing CD15 were previously reported [26].

Phenomenon of lineage infidelity when monoclonally rearranged IG and TR genes coexist in the same cell has been well recognized. Its frequency varies from 5 to $10 \%$ of welldifferentiated B cell neoplasms, e.g., B cell chronic lymphocytic leukemia to $70 \%$ of less-differentiated B cell neoplasms, e.g., B acute lymphoblastic leukemia/lymphoma. Several studies of relapsing lymphomas and B acute lymphoblastic leukemias [27-33] revealed that the monoclonal rearrangements of the IGH and, to a lesser extent, TR genes can be 
capricious. These genes can disappear at relapse, and the primary neoplasms can be overgrown by subclones with divergent rearrangement patterns. Similar changes likely took place during the course of transformation of our case.

Although gradual transformation of B cell lymphoma into $\mathrm{T}$ cell lymphoma is a rarity, this phenomenon is likely to be of clinical significance in individual cases.

Conflict of interest The authors declare that they have no conflict of interest.

\section{References}

1. York JC II, Cousar JB, Glick AD, Flexner JM, Stein R, Collins RD (1985) Morphologic and immunologic evidence of composite Band T-cell lymphomas. A report of three cases developing in follicular center cell lymphomas. Am J Clin Pathol 84:35-43

2. Delabie J, Greiner TC, Chan WC, Weisenburger DD (1996) Concurrent lymphocyte predominance Hodgkin's disease and Tcell lymphoma. A report of three cases. Am J Surg Pathol 20: 355-362

3. Niedobitek G, Baumann I, Brabletz T, Lisner R, Winkelmann C, Helm G, Kirchner T (2000) Hodgkin's disease and peripheral T-cell lymphoma: composite lymphoma with evidence of Epstein-Barr virus infection. J Pathol 191:394-399

4. Hirose Y, Fukushima T, Masaki Y, Shimoyama K, Karasawa H, Ogawa N, Wano Y (2004) Epstein-Barr virus-associated composite lymphoma composed of peripheral T-cell lymphoma and an anaplastic variant of a diffuse large B-cell type of non-Hodgkin's lymphoma and strongly expressing p53 protein. Int J Hematol 79:260265

5. Kaleem Z, McGuire MH, Caracioni AC, Leonard RL, Pathan MH, Lessmann EA, Chan WC (2005) Composite B-cell and T-cell nonHodgkin lymphoma of the tibia. Am J Clin Pathol 123:215-221

6. Sanchez S, Holmes H, Katabi N, Newman J, Domiatti-Saad R, Stone M, Netto G (2006) Composite lymphocyte-rich Hodgkin lymphoma and peripheral T-cell lymphoma associated with Epstein-Barr virus: a case report and review of the literature. Arch Pathol Lab Med 130:107-112

7. Demurtas A, Aliberti S, Bonello L, Di Celle PF, Cavaliere C, Barreca A, Novero D, Stacchini A (2011) Usefulness of multiparametric flow cytometry in detecting composite lymphoma: study of 17 cases in a 12-year period. Am J Clin Pathol 135:541555

8. Wang E, Papavassiliou P, Wang AR, Louissaint A Jr, Wang J, Hutchison CB, Huang Q, Reddi D, Wei Q, Sebastian S, Rehder C, Brynes R, Siddiqi I (2014) Composite lymphoid neoplasm of B-cell and T-cell origins: a pathologic study of 14 cases. Hum Pathol 45:768-784

9. Campidelli C, Sabattini E, Piccioli M, Rossi M, De Blasi D, Miraglia E, Rodriguez-Abreu D, Franscini LL, Bertoni F, Mazzucchelli L, Cavalli F, Zucca E, Pileri SA (2007) Simultaneous occurrence of peripheral T-cell lymphoma unspecified and B-cell small lymphocytic lymphoma. Report of 2 cases. Hum Pathol 38:787-792

10. Furlan A, Pietrogrande F, Marino F, Menin C, Polato G, Vianello F (2008) Sequential development of large B cell lymphoma in a patient with peripheral T-cell lymphoma. Haematologica 93:e6-e8

11. van Dongen JJ, Langerak AW, Bruggemann M, Evans PA, Hummel M, Lavender FL, Delabesse E, Davi F, Schuuring E, Garcia-Sanz R, van Krieken JH, Droese J, Gonzales D, Bastard C, White HE, Spaargaren M, Gonzalez M, Parreira A, Smith JL, Morgan GJ, Kneba M, Macintyre EA (2003) Design and standardization of PCR primers and protocols for detection of clonal immunoglobulin and T-cell receptor gene recombinations in suspect lymphoproliferations: report of the BIOMED-2 Concerted Action BMH4-CT98-3936. Leukemia 17:2257-2317

12. Altschul SF, Gish W, Miller W, Myers EW, Lipman DJ (1990) Basic local alignment search tool. J Mol Biol 215:403-410

13. Ye J, Ma N, Madden TL, Ostell JM (2013) Ig-BLAST: an immunoglobulin variable domain sequence analysis tool. Nucleic Acids Res 41(Web Server issue):W34-W40

14. Williams C, Ponten F, Moberg C, Soderkvist P, Uhlen M, Ponten J, Sitbon G, Lundeberg J (1999) A high frequency of sequence alterations is due to formalin fixation of archival specimens. Am J Pathol 155:1467-1471

15. Spencer DH, Sehn JK, Abel HJ, Watson MA, Pfeifer JD, Duncavage EJ (2013) Comparison of clinical targeted nextgeneration sequence data from formalin-fixed and fresh-frozen tissue specimens. J Mol Diagn 15:623-633

16. Rossi D, Berra E, Cerri M, Deambrogi C, Barbieri C, Franceschetti S, Lunghi M, Conconi A, Paulli M, Matolcsy A, Pasqualucci L, Capello D, Gaidano G (2006) Aberrant somatic hypermutation in transformation of follicular lymphoma and chronic lymphocytic leukemia to diffuse large B-cell lymphoma. Haematologica 91: 1405-1409

17. Cobaleda C, Jochum W, Busslinger M (2007) Conversion of mature $\mathrm{B}$ cells into $\mathrm{T}$ cells by dedifferentiation to uncommitted progenitors. Nature 449:473-477

18. Barneda-Zahonero B, Roman-Gonzalez L, Collazo O, Mahmoudi T, Parra M (2012) Epigenetic regulation of B lymphocyte differentiation, transdifferentiation, and reprogramming. Comp Funct Genomics 2012:564381

19. McManus S, Ebert A, Salvagiotto G, Medvedovic J, Sun Q, Tamir I, Jaritz M, Tagoh H, Busslinger M (2011) The transcription factor Pax 5 regulates its target genes by recruiting chromatin-modifying proteins in committed B cells. EMBO J 30:2388-2404

20. Feldman AL, Minniti C, Santi M, Downing JR, Raffeld M, Jaffe ES (2004) Histiocytic sarcoma after acute lymphoblastic leukemia: a common clonal origin. Lancet Oncol 5:248-250

21. Feldman AL, Arber DA, Pittaluga S, Martinez A, Burke JS, Raffeld M, Camos M, Warnke R, Jaffe ES (2008) Clonally related follicular lymphomas and histiocytic/dendritic cell sarcomas: evidence for transdifferentiation of the follicular lymphoma clone. Blood 111: 5433-5439

22. Kumar R, Khan SP, Joshi DD, Shaw GR, Kettering RP, Feldman AL (2011) Pediatric histiocytic sarcoma clonally related to precursor B-cell acute lymphoblastic leukemia with homozygous deletion of CDKN2A encoding p161NK4A. Pediatr Blood Cancer 56:307310

23. Wang E, Papalas J, Hutchinson CB, Kulbacki E, Huang Q, Sebastian S, Rehder C, Silbermins D, Moore J, Datto M (2011) Sequential development of histiocytic sarcoma and diffuse large B-cell lymphoma in a patient with a remote history of follicular lymphoma with genotypic evidence of a clonal relationship: a divergent (bilineal) neoplastic transformation of an indolent B-cell lymphoma in a single individual. Am J Surg Pathol 35:457-463

24. Chen X, Rutledge JC, Wu D, Fang M, Opheim KE, Xu M (2013) Chronic myelogenous leukemia presenting in blast phase with nodal, bilineal myeloid sarcoma and T-lymphoblastic lymphoma in a child. Pediatr Dev Pathol 16:91-96

25. West DA, Dogan A, Quint PS, Tricker-Klar ML, Porcher J, Ketterling R, Law ME, PcPhail ED, Viswanatha DS, Kurtin PJ, Dao LN, Ritzer RD, Nowakowski GS, Feldman AL (2013) Clonally related follicular lymphomas and Langerhans cell neoplasms. Am J Surg Pathol 37:978-986 
26. Gorczyca W, Tsang P, Liu Z, Wu Z, Wu CD, Dong HY, Goldstein M, Cohen M, Cohen P, Gangi M, Weisberger J (2003) CD30positive T-cell lymphomas co-expressing CD15: an immunohistochemical analysis. Int J Oncol 22:319-324

27. de Jong D, Voetdijk BM, van Ommen GJ, Kluin PM (1989) Alterations in immunoglobulin genes reveal the origin and evolution of monotypic and bitypic B cell lymphomas. Am J Pathol 134: 1233-1242

28. Lindh J, Linderholm B, Lindstrom A, Hagberg H, Sundstrom C, Roos G (1994) Multiclonality and altered RFLP patterns for immunoglobulin heavy-chain and T-cell receptor genes in relapsing lymphomas. Ann Oncol 5:75-78

29. Garcia MJ, Martinez-Delgado B, Granizo JJ, Benitez J, Rivas C (2001) IgH, TCR- $\gamma$, and TCR- $\beta$ gene rearrangement in $80 \mathrm{~B}$ - and T-cell non-Hodgkin's lymphomas: study of the association between proliferation and the so-called "aberrant" patterns. Diagn Mol Pathol 10:69-77

30. Li AH, Rosenquist R, Forestier E, Lindh J, Roos G (2001) Detailed clonality analysis of relapsing precursor B acute lymphoblastic leukemia: implications for minimal residual disease detection. Leuk Res 25:1033-1045

31. Szczepanski T, Willemese MJ, Brinkhof B, van Wering ER, van der Burg M, van Dongen JJ (2002) Comparative analysis of Ig and TCR gene rearrangements at diagnosis and at relapse of childhood precursor-B-ALL provides improved strategies for selection of stable PCR targets for monitoring of minimal residual disease. Blood 99:2315-2323

32. Li A, Zhou J, Zuckerman D, Rue M, Dalton V, Lyons C, Silverman LB, Sallan SE, Gribben JG (2003) Sequence analysis of clonal immunoglobulin and T-cell receptor gene rearrangements in children with acute lymphoblastic leukemia at diagnosis and at relapse: implications for pathogenesis and for the clinical utility of PCRbased methods of minimal residual disease detection. Blood 102: $4520-4526$

33. Bagg A (2006) Immunoglobulin and T-Cell receptor gene rearrangements: minding your B's and T's in assessing lineage and clonality in neoplastic lymphoproliferative disorders. J Mol Diagn $8: 426-429$ 